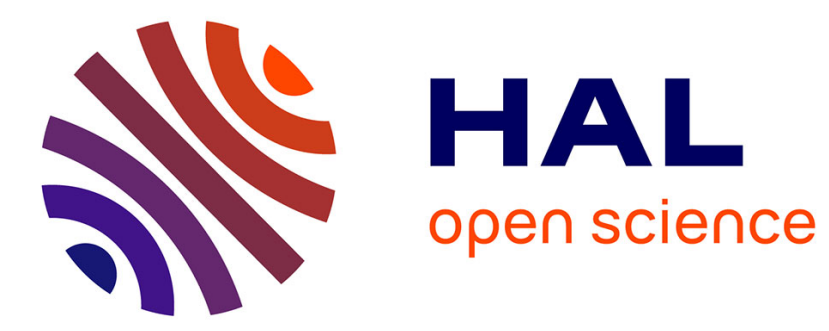

\title{
Un manche d'éventail en ivoire à Argentomagus (Saint-Marcel, Indre)
}

Gérard Coulon, Isabelle Fauduet

\section{To cite this version:}

Gérard Coulon, Isabelle Fauduet. Un manche d'éventail en ivoire à Argentomagus (Saint-Marcel, Indre). Gallia - Fouilles et monuments archéologiques en France métropolitaine, 1991, 48, pp.337-343. 10.3406/galia.1991.2924. hal-01915171

\section{HAL Id: hal-01915171 \\ https://hal.science/hal-01915171}

Submitted on 19 Jan 2020

HAL is a multi-disciplinary open access archive for the deposit and dissemination of scientific research documents, whether they are published or not. The documents may come from teaching and research institutions in France or abroad, or from public or private research centers.
L'archive ouverte pluridisciplinaire $\mathbf{H A L}$, est destinée au dépôt et à la diffusion de documents scientifiques de niveau recherche, publiés ou non, émanant des établissements d'enseignement et de recherche français ou étrangers, des laboratoires publics ou privés.

\section{(ㅇ)(1) $\$$}

Distributed under a Creative Commons Attribution - NonCommercial - NoDerivatives| 4.0 


\title{
Un manche d'éventail en ivoire à Argentomagus (Saint-Marcel, Indre)
}

\author{
par Gérard COULON et Isabelle FAUDUET
}

( - Un manche d'éventail en ivoire a été découvert à Argentomagus au cours des fouilles pratiquées à l'emplacement du nouveau musée archéologique. Cet accessoire, décrit par les auteurs anciens mais rarement retrou-

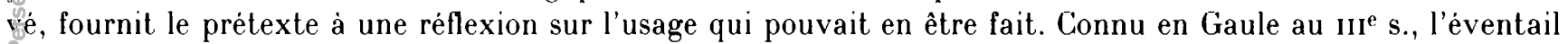
plissé circulaire est encore attesté au début du Moyen Age comme instrument de culte. N'était-il pas non plus associé, dans certains cas, à un rituel funéraire comme en témoignent les quelques exemplaires recueillis dans des sépultures et les représentations figurées du monde étrusque, hellénistique et romain?

An ivory stick from a fan has been discovered at Argentomagus (St Marcel, Indre) during the excavation carried on the place of the new archaeological Museum. This accessory is described by ancient writers but scarcely ever found so it gives the opportunity to raise the question of its function. Known in Gaul through the 3rd century AD, the round plaited fan still occurs during the early Middle Ages, as part of the ritual equipment. Was it also related, under parlicular circumstances, to burial customs for which evidence is illustrated by a few examples in burials and by representations from the Etruscan, Hellenistic and Roman world?

Mots clés : éventail, ivoire, rituel funéraire, Gallo-romain, Argentomagus, Saint-Marcel, Indre. 

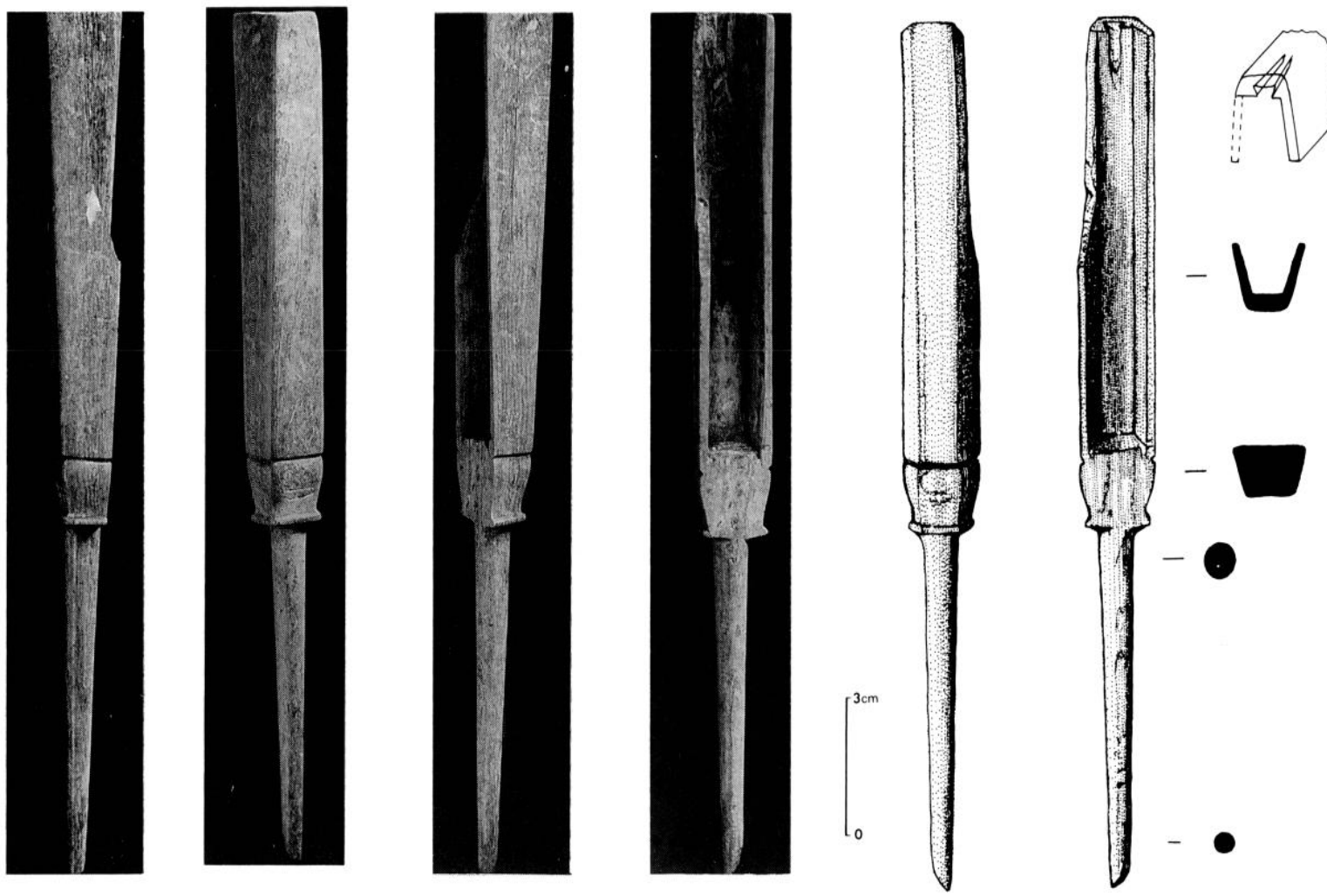

Fig. 1 - Manche d'éventail en ivoire d'Argentomagus (Saint-Marcel, Indre).

Les fouilles effectuées de 1983 à 1987 préalablement à la construction du nouveau musée archéologique d'Argentomagus, à Saint-Marcel, ont permis de mettre au jour un quartier de l'agglomération gallo-romaine ${ }^{1}$. Ce quartier était implanté au croisement de deux rues orthogonales aménagées après le comblement partiel de l'ancien fossé de l'oppidum.

Parmi l'abondant mobilier archéologique recueilli au cours de ces cinq campagnes de fouilles, figure un manche d'éventail en ivoire ${ }^{2}$. Un objet que nos recherches nous incitent à considérer comme extrêmement rare en Gaule.

1 Sur ces fouilles, A. Frandìre, Y. de Kisch, Informations archéologiques, Circonscription du Centre, Gallia, 42, 1984, p. 322-323; - G. Covıon, Saint-Marcel (Indre), Argentomagus, résultats 1982-1984, Les Mersans, Revue Archéologique du Centre de la France (RACF), 24, 1, 1985, p. 115-116; - Saint-Marcel (Indre), Argentomagus, sauvetage programmé à l'emplacement du futur musée, campagne $1985, R A C F, 25,1$, 1986, p. 107-110 et id., campagnes 1986-1987, RACF, 27, 1, 1988, p. 131-133.

2 L'objet provient du niveau le plus riche du secteur 204 (couche 204005). De texture homogène, ce niveau est datable de la fin du $\mathrm{II}^{\mathrm{P}} \mathrm{s}$. avec des éléments des $\mathrm{I}^{\mathrm{er}}$ et $\mathrm{II}^{\mathrm{P}} \mathrm{s}$. (les plus précoces sont des fragments de Drag. 29 et une monnaie de Claude)

\section{Description}

Ce manche d'èventail (fig. 1) se présente sous la forme d'un objet allongé (longueur : $18,8 \mathrm{~cm}$ ), relativement étroit (largeur maximale : $1,8 \mathrm{~cm}$ ), compose de trois parties :

- une partie effilée dont l'extrémité inférieure est brisée de sorte que sa longueur en l'état n'est que de $7,7 \mathrm{~cm}$. De section circulaire, son diamètre va croissant, passant de 0,4 à $0,8 \mathrm{~cm}$. C'est le manche proprement dit, c'est-à-dire la partie tenue dans la main ;

- une partie médiane de section trapézoïdale (grande base : $1,7 \mathrm{~cm}$, petite base : $1,1 \mathrm{~cm}$, hauteur : $1,1 \mathrm{~cm})$. Cette partie intermédiaire moulurée est longue de $1,7 \mathrm{~cm}$;

- une partie évidée, longue de $9,4 \mathrm{~cm}$, qui offre une section en $\mathrm{U}$ à fond plat et à bords légèrement évasés. La hauteur de ses côtés croît régulièrement, passant de 1,1 à $2,0 \mathrm{~cm}$. Quant à la profondeur de la gorge, elle passe de ce fait, de 0,8 à $1,5 \mathrm{~cm}$. L'un des côtés est endommagé par une cassure assurément ancienne puisque la patine de la tranche y est identique à celle de l'ensemble de l'objet. Cette partie évidée se termine par une échancrure en forme de queue d'aronde, longue de $1,1 \mathrm{~cm}$ et large de $0,5 \mathrm{~cm}$. 


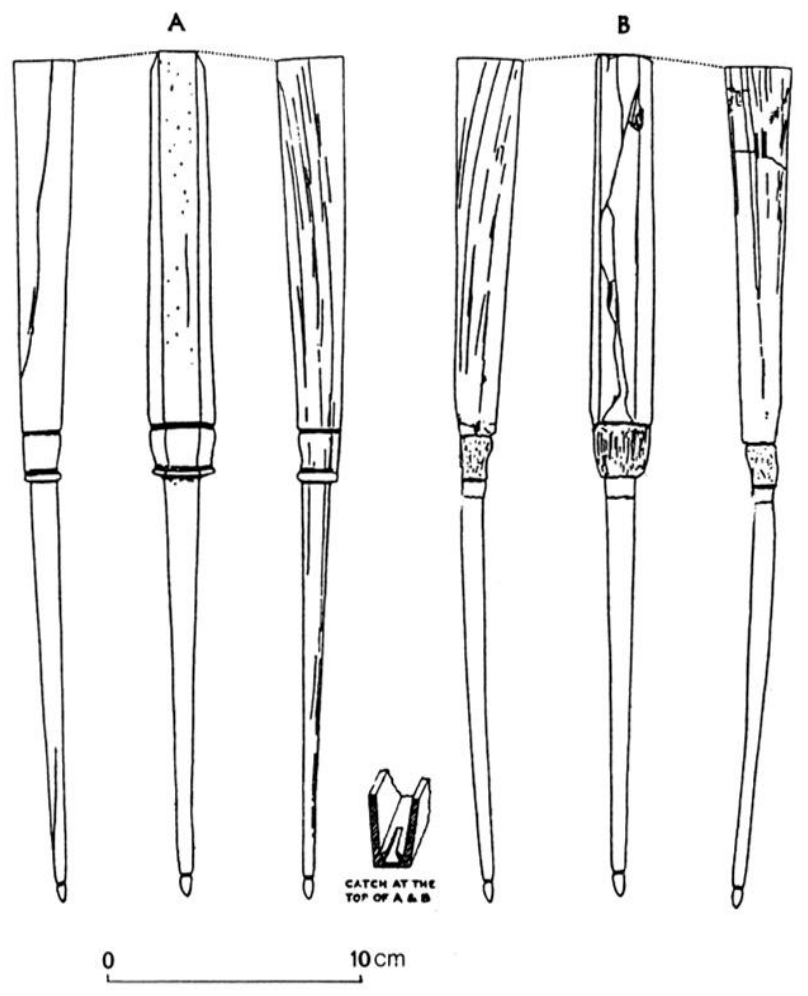

Fig. 2 - Les deux manches d'éventail d'Eburacum (York, Grande-Bretagne) (d'après I. Richmond).

Ce manche d'éventail a été façonné dans de l'ivoire d'éléphant. Il a été taillé dans la pointe de la défense et l'examen montre que la partie évidée était proche du coeur de celle-ci tandis que la partie "dorsale" était voisine de sa surface ${ }^{3}$.

\section{Interprétation}

Seul un des deux éléments du manche nous est parvenu. Il en fallait en effet deux semblables pour qu'ils fussent fonctionnels. Quant à la feuille, elle pouvait être faite de fines lamelles de bois assemblées, de peau ou d'étoffe tendues sur une armature de baguettes.

En position fermée, on ne voyait de l'éventail que ses deux manches placés en vis-à-vis. La feuille était alors repliée dans la partic ćvidéc de l'instrument. Pour ouvrir l'éventail, on déployait la feuille et on plaçait les deux manches dos à dos. L'objet était alors tenu dans la main par les deux parties effilées serrées l'une contre l'autre. L'échancrure en

3 Diagnostic de François Poplin à qui nous adressons nos vifs remerciements. queue d'aronde permettait évidemment de maintenir les deux extrémités de la feuille fixées au manche. Il est raisonnable de penser que cette feuille était collée sur toute sa largeur dans son logement afin de lui assurer une fixation efficace.

\section{Éléments de comparaison}

Les découvertes de ce type d'instrument en ivoire restent exceptionnelles puisque cinq seulement nous sont connues pour tout l'Occident romain.

York (Grande-Bretagne). L'une des nécropoles de l'antique Eburacum a livré deux manches d'un éventail pliant, tout à fait semblables à celui d'Argentomagus mais longs de $32,0 \mathrm{~cm}$ (fig. 2). On observe là aussi les traces de l'encoche qui servait à l'attache des lamelles. Ils ont été recueillis dans une sépulture féminine à inhumation datable du III $^{\mathrm{e}} \mathrm{s}$. La reconstitution proposée (fig. 3) a èté réalisée au moyen de lamelles de bois (Eburacum..., 1962, p. 82, pl. 71).

Cologne (Allemagne). Au cours de ses recherches sur la tabletterie de la Bretagne romaine, S. Greep signale un manche d'éventail comparable à ceux d'York. Ce manche inédit est conservé au RömischGermanische Museum de Cologne. Bien qu'incomplet, celui-ci présente un décor de paons ${ }^{4}$.

Selongey (France, Côte-d'Or). Un manche d'éventail provient d'une cave de la villa galloromaine des Tuillières. Il a été découvert avec des objets d'ivoirc, divers accessoires dont les éléments d'un coffre (III $\mathrm{e}$.) et de quelques armes (casque, glaive, pilum, cuirasse) ${ }^{5}$.

Arles (France, Bouches-du-Rhône). Les deux manches d'un éventail (longueur : $29,0 \mathrm{~cm}$ ) ont été recueillis dans une sépulture féminine du $I^{\circ} \mathrm{s}$. Des traces d'or ont été observées sur ces manches conservés au musée Réattu d'Arles ${ }^{6}$.

4 GREEP, 1983, p. 80. Nous sommes reconnaissants à l'auteur de nous avoir communiqué des renseignements sur ce fragment de Cologne.

5 Ce mobilier pourrait appartenir à un ancien militaire installé dans une exploitation agricole, cf. Il élail une fois la Côte-d'Or, vingt ans de recherches archéologiques, Musèe archéologique de Dijon, Paris, Éd. Errance, 1990, p. 95-97 (renseignement donné par $\mathrm{A}$. Ruellet et $\mathrm{S}$. Deyts).

6 Inv. IRP 891254831 et IRP 891255831 . Précision apportée par Claude Sintès. L'objet est illustré dans Carnets de fouilles d'une presqu'île, Revue d'Arles, 2 , Arles, Salles romanes du cloître, juin-octobre 1990 (catalogue d'exposition), p. 38 , 39 . 
Fig. 3 -

a. essai de reconstitution de l'éventail d'Eburacum; b. reconstitution graphique de l'éventail d'Eburacum (York. (irande-Bretagne) en position ouverte et fermée (d apres I. Richmond).
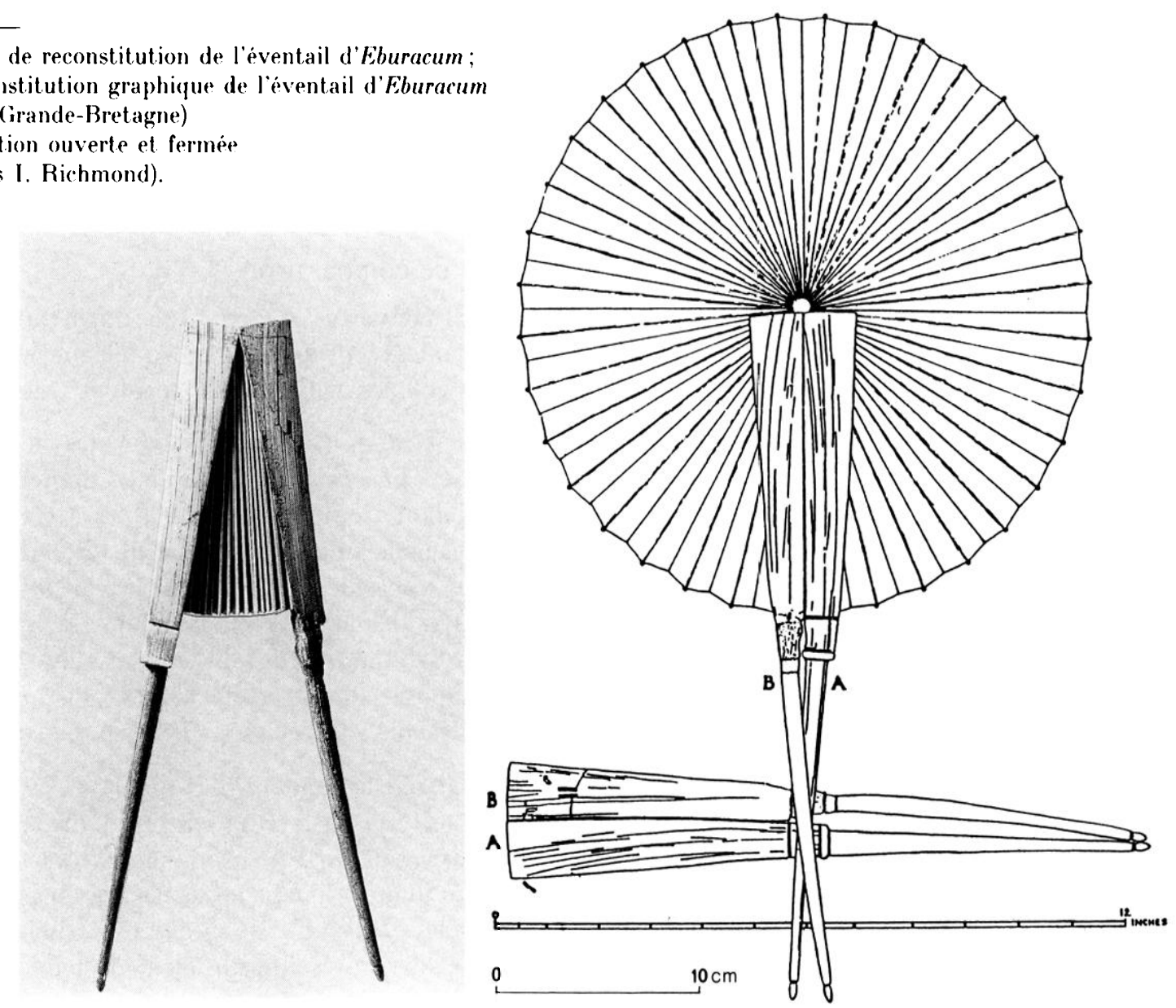

Quelques documents figurés montrent des éventails circulaires et plissés :

- sur une stèle funéraire du musée Rolin d'Autun (provenance inconnue, inv. M.L. 142), on voit une femme en buste tenant de la main gauche un éventail ouvert et de la main droite un gobelet (fig. 4). On reconnaît les deux manches parallèles et les lamelles disposées en rayons. Sur une autre stèle d'Autun (inv. M.L. 181), l'objet circulaire que tient la défunte est plus difficile à identifier et évoque davantage un miroir (Aulun-Augustodunum, 1987, p. 263$)$;

- un relief du Carlisle Museum and Art Gallery (Grande-Bretagne), trouvé à Murrel Hill, Cumberland, représente une femme assise agitant de la main droite un large éventail (fig. 5). Un enfant se tient près d'elle et deux lions ornent le fronton. Cette stèle est datée des II $^{\mathrm{e}}-\mathrm{III}^{\mathrm{P}} \mathrm{s}$. (Liversidge, 1973, pl. 26);

- sur un vase d'Arezzo conservé au Museum of Fine Arts de Boston (États-Unis), on aperçoit une femme agitant un éventail du même type, marchant derrière un personnage qui tient un parasol (fig. 6). Ils font partie d'une scène qui représente un char tiré par un centaure et sur lequel sont étendus la reine Omphale et Héraklès déguisé en femme (Chapot, 1918, p. 214) ${ }^{7}$;

- l'éventail plissé circulaire existe encore au début du Moyen Age. Quelques lieux saints en conservent. L'exemplaire de Monza (Italie), daté du

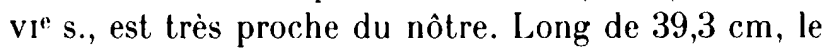
manche est également constitué d'une partie cylindrique séparée d'un boîlier rectangulaire par une moulure. Ce boîtier est orné de motifs végétaux. Cet éventail porte un anneau à sa base permettant, par exemple, de le suspendre à la ceinture (De Vere Green, 1975, p. 87).

7 Description reprise plus récemment dans C. VI:Rmrule, Greek, Etruscan and Roman Arl, Classical Collection of Museum of Fine Arts, Boston, 1963, p. 267. 


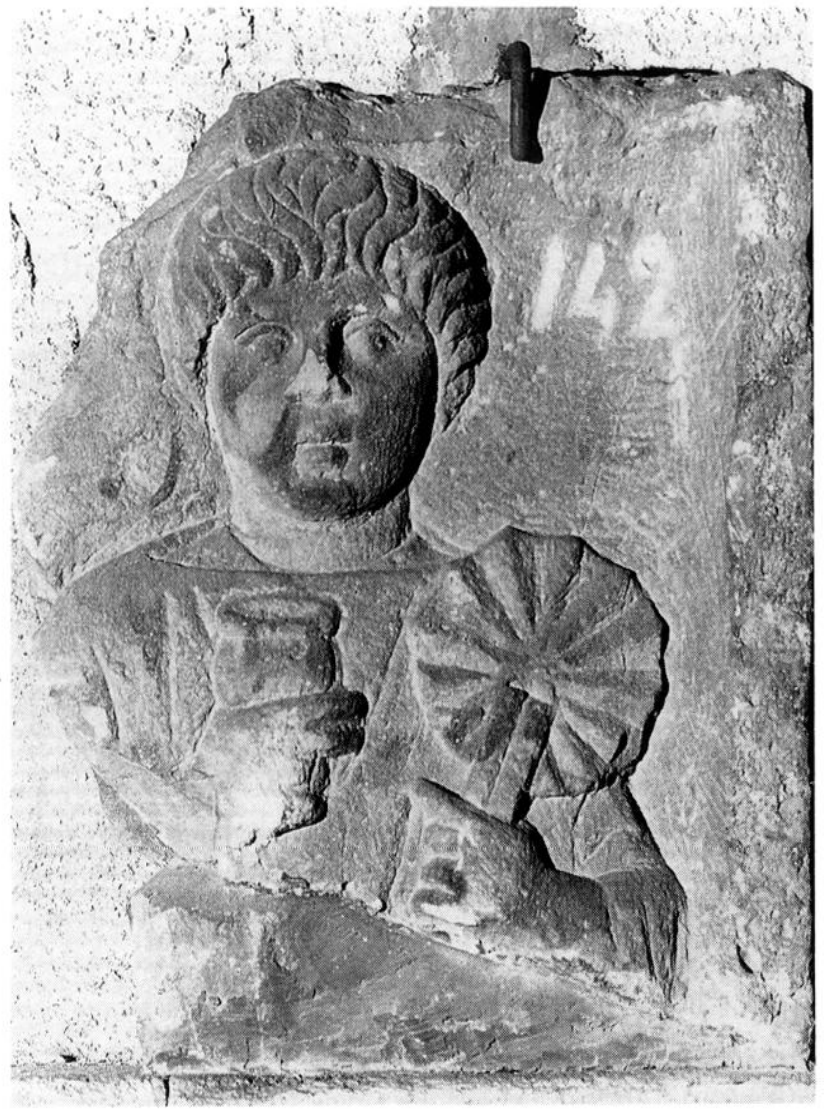

Fig. 4 - Stèle funéraire d'une femme tenant un gobelet et un eventail. Hauteur : $45,0 \mathrm{~cm}$; largeur : $40,0 \mathrm{~cm}$; épaisseur : $21,0 \mathrm{~cm}$ (Musée Rolin, Autun, Inv. M.L. 142). (Espérandieu, Recueil général des bas-reliefs, statues et bustes de la Gaule romaine, III, Lyonnaise, $1^{\text {re }}$ partie, p. $\left.81-82, n^{\prime \prime} 1880\right)$.

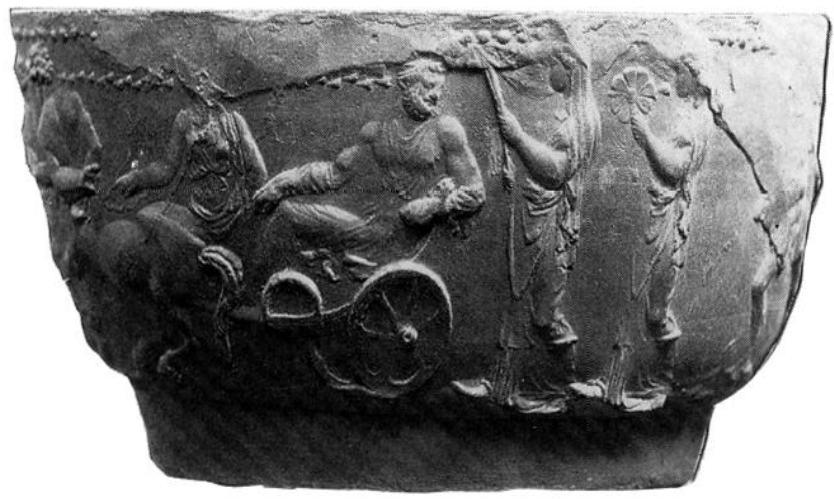

Fig. 6 - Fragment de vase d'Arezo montrant à droite une femme tenant un éventail circulaire plissé. (Museum of Fine Arts, Boston. États-Linis. Inv. no 98 870).

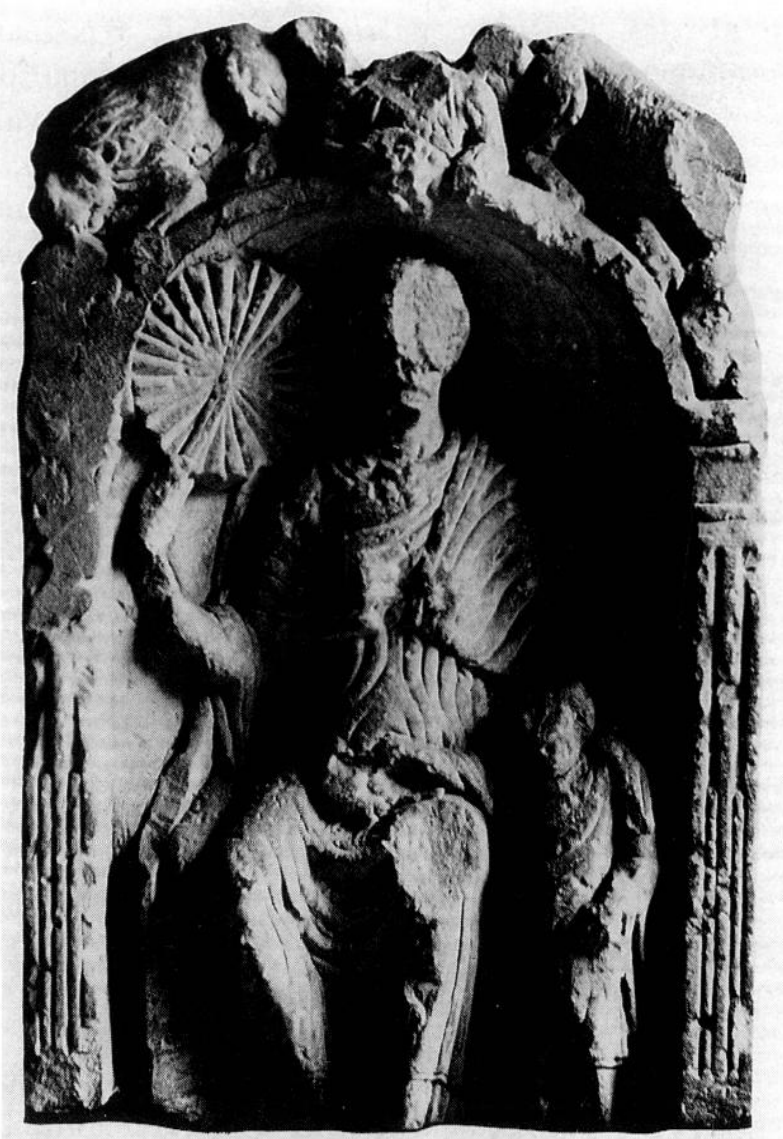

Fig. 5 - Stèle funéraire d'une femme assise agitant un éventail de la main droite. Hauteur : 1,30 m. (Carlisle Museum and Art Gallery, Inv. ().M. 374).

\section{L'éventail dans l'antiquité romaine}

Si dans l'Antiquité romaine l'éventail a revêtu des formes diverses, toutes ses variantes ressortissaient de deux types principaux : l'éventail plissé et circulaire se refermant sur lui-même et l'écran à manche.

Le premier vient d'être décrit avec les exemplaires présentés ci-dessus et les figurations des musées d'Autun, Carlisle et Boston. C'est selon toute vraisemblance un éventail de ce type, réalisé en fines lamelles de bois, qu'évoque Ovide lorsque dans l'Arl d'aimer (I, 161), il désigne cet accessoire par le mot labella.

L’écran à manche le plus recherché était réalisé à laide de plumes de paon insérées à l'extrémité du manche ou emboitées dans une monture ouvragée de bois ou d'ivoire. Martial (Epigrammes, XIV, 67), Claudien (Eutrop., I, 108) et Properce (II, 18. 50) 
citent des pavonina flabella que l'on reconnait notamment sur une peinture du Musée national de Naples provenant d'Herculanum ${ }^{8}$. L'écran pouvait aussi être réalisé au moyen de plumes d'autruche, à partir de la toison soyeuse du yack, en bois mince peint - Martial mentionne un éventail vert (Epigrammes, III, 82, 10) - ou en paille tressée.

Un manche du musée de Split (Yougoslavie) se rattache à ce type. Cylindrique, il est en os ciselé ; on distingue surtout, du décor, la tête d'une femme dont le corps est entouré d'un serpent et une série de signes hiéroglyphiques. Ce manche devait maintenir une touffe de plumes, indique Crochet qui a décrit l'objet (Crochet, 1888, p. 31).

\section{Les fonctions de l'éventail}

Les exemplaires décrits dans cet article sont très semblables, ce qui laisse suggérer un lieu ou une aire de fabrication unique, compte tenu en particulier de leur caractère luxueux. S'il apparaît comme à peu près certain que ces instruments en ivoire furent importés au cours du $\mathrm{III}^{\mathrm{e}} \mathrm{s}$., aucun indice ne permet actuellement de préciser leur lieu de production.

On peut raisonnablement supposer que la plupart des éventails étaient réalisés en bois, ce qui justifierait la rareté des trouvailles. Rareté qui pourrait également s'expliquer par un usage peu répandu en Gaule et dans les contrées septentrionales.

Quelle était la destination de l'éventail d'Argentomagus? On sait qu'il avait sa place chez les dames romaines. En témoignent les allusions des auteurs anciens, Martial (Epigrammes, III, 82, 10), Térence (Eunuque, III, 595) et Properce (II, 24, 11). Il apportait au visage un peu de fraîcheur pendant la promenade, au spectacle, pendant le repos ou dans l'atmosphère étouffante des thermes. Des esclaves, hommes ou femmes, étaient parfois chargés de cette fonction - fabrarii ou flabelliferae - (Claudien, Eutrop. I, 108-109, Plaute, Trinum, II, 251). L'èventail pouvait également faire office de chasse-mouches (muscarium).

Il servait aussi dans certains actes religieux. C'est ainsi qu'une peinture d'Herculanum ${ }^{9}$ montre un prêtre agitant un éventail triangulaire au-dessus de la flamme d'un autel. W. Deonna le cite parmi les ex-voto rencontrés dans des sanctuaires grecs ${ }^{10}$.

8 Daremberg et Saglio, 1877, fig. 3071, p. 1151.

9 Cf. note 8.

10 W. Dronna, Le mobilier délien, Exploration archéologique de Délos, École Française d'Athènes, XVIII, Paris, 1938 , p. 273.
D'après L. Crochet, le décor du manche de Split laisserait à penser que des prêtres d'Isis utilisaient un éventail pendant des cérémonies religieuses.

Si nous ne disposons pas de témoignages concernant les lieux de culte gallo-romains, en revanche, le flabellum est attesté comme accessoire cultuel dans l'Église primitive, plus particulièrement dans les provinces orientales. Il est agité pendant les processions et célébrations, notamment par les diacres pour empêcher les mouches de tomber dans le calice ${ }^{11}$. Est-ce un emprunt au rituel des cultes orientaux ${ }^{12}$ ? Rappelons que les manches d'York et de Cologne proviennent de sites où un mithraeum et des cultes orientaux sont attestés. La présence de deux lions sur la stèle de Carlisle est inhabituelle. Ces accessoires ont-ils appartenu à des étrangers, à des dévots de cultes orientaux?

L'éventail a-t-il par ailleurs un rôle funéraire? Il figure sur des stèles avec d'autres accessoires qui évoquent le thème du voyage vers le monde des morts (miroir, gobelet, patère...). Si les représentations d'une défunte tenant un éventail sont répandues sur quelques variétés de vases grecs et étrusques ${ }^{13}$ et sur des stèles hellénistiques ${ }^{14}$, elles sont particulièrement saisissantes sur des urnes cinéraires étrusques des $\mathrm{II}^{\mathrm{e}}$ et $\mathrm{I}^{\text {er }} \mathrm{s}$. avant $\mathrm{J}$.-C. La femme est représentée étendue sur le coude, tenant bien en évidence soit un éventail à feuilles ou à plumes très orné, soit une phiale ${ }^{15}$. L'exemple très réaliste de

11 F. Cabrol, Dictionnaire des antiquités chrétiennes et de liturgie, vol. 2, Paris, 1923, p. 1614; - J. Leroy, Un flabellum syriaque daté du deir Sauriani, Egypte, Cahiers de Mariémont, 5-6, 1974/1975, p. $31 s q$.

12 De Vere Green, 1975 , p. $80 ;-$ A. Butler, The Ancient Coptic Churches of Egypt, II, Oxford, 1970, p. 46-53.

13 L'éventail figure parmi les objets à caractère rituel dans des scènes funéraires : citons notamment des hydries, pelikés ou des onochoés de quelques peintres d'Apulie du IV" $\mathrm{s}$. avant $J .-C$., vases sur lequels une défunte ou sa servante est représentée tenant un éventail devant un autel ou dans un naiskos, cf. A.D. Trendal.., A. Cambitoglou, The Red-figured Vases of Apulia, II, Oxford monographies on Classical Archaeology, 1982, pl. 201, 204, 215, 216, 217, 326sq. et p. $541,542,868$ et 869 .

L'accès à la base de données des Antiquités grecques et romaines du Centre Charbonneaux au Musée du Louvre a facilité cette recherche des documents de comparaison.

14 Particulièrement sur des stèles du $\mathrm{II}^{\mathrm{e}} \mathrm{s}$. avant J.-C. en Asie Mineure : E. Pfunt, H. Mörıus, Die ostgriechischen Grabreliefs, I, 1977, Mayence, pl. 60, 91, 112 (stèles à naiskos en marbre).

15 Urne Volterrane $1-I$ complessi tombali, Corpus delle urne etrusche di eta ellenestica 1. Centro Di, Florence, 1975 (tombes 16, 122, 231, 259, 283, 288) et idem 2 - il museo Guarnacci, 1977 (tombes 80, 86, 113, 129, 238, 240). 
Volterra, reproduit ici (fig. 7), est d'autant plus marquant que, sur la cuve de l'urne, on distingue un carpenlum gaulois très évocateur ${ }^{16}$. L artisan n'a pas sculpté cet objet comme un simple accessoire de la vie quotidienne mais comme un instrument prophylactique, propre peut-être à chasser les mauvais esprits dans l'Au-delà?

Rappelons que les éventails d'York et d'Arles ont été recueillis dans un contexte funéraire. Sont-ils seulement de simples objets familiers déposés en tant que tels dans une tombe ou traduisent-ils une pratique funéraire encore mal connue en Occident alors qu'elle est mieux attestée dans le monde méditerranéen? Plus tard, pour les chrétiens du Moyen Age, agiter l'éventail servait, de la même façon, à éloigner les démons (De Vere Green, 1975, p. 83).

Les stèles d'Autun et de Carlisle sont-elles les modestes témoins d'une semblable coutume?

\section{Gérard Coulon et Isabelle: Fauduet}

16 F. Villard (ed.), Aspects de l'Art Étrusque dans les collections du musee du Louvre, Paris, s.d., p. 40. Nous remercions Marie-Françoise Briguet de nous avoir autorisés a publier ce document.

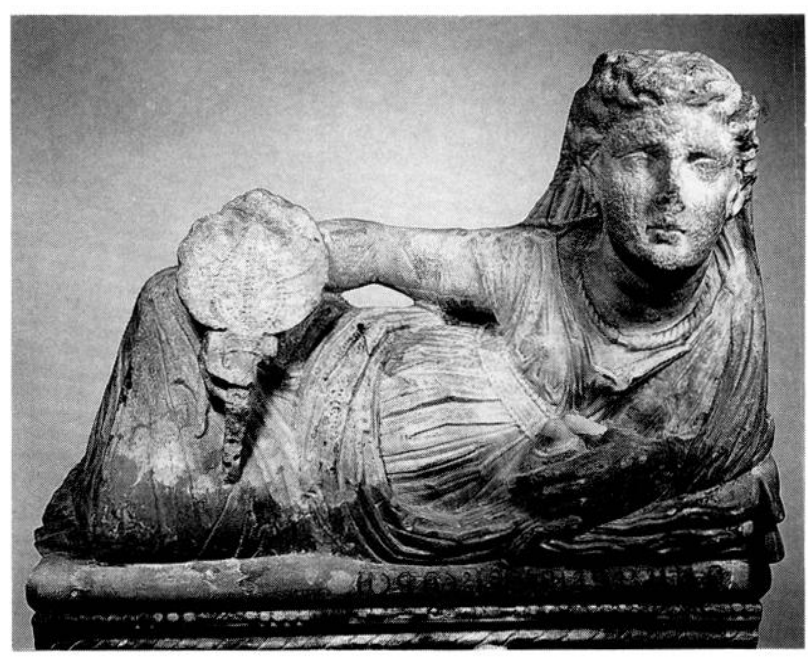

Fig. 7 - Lrne cinéraire étrusque de Volterra de la fin du II $^{*}$ s. - début du $\mathrm{I}^{\mathrm{er}} \mathrm{s}$. avant J.-C. Hauteur : 0,84 m. (Musée du Louvre, Inv. M.A. 2 357).

Origine des documents.

L. Meslin (fig. 1, dessin); R. Gesell (fig. 1, photographie): Yorkshire Museum (fig. 3); G. Varlez (fig. 4); Carlisle Museum and Art Gallery (fig. 5): Museum of Fine Arts (fig. 6): Musée du Louvre (fig. 7).

\section{BIBLIOGRAPHIE}

\section{Autun-Augustodunum}

Aulun-Augustodunum, Capitale des Eduens, Ville d'Autun, Musée Rolin, 1987, 411 p.

\section{Chapot V.}

1918 : Séance du 16 octobre, Bulletin de la Société Nationale des Anliquaires de France, p. 214.

\section{Crochet $\mathbf{L}$.}

1888 : La toilette chez les Romains au temps des empereurs, Lyon, 71 p.

\section{Daremberg C., Saglio E.}

1877 : Dictionnaire des antiquités grecques et romaines. flabellum (G. Fougères), vol. 4, p. 1149-1152.

De Vere Green B.

1975: A Collector's Guide to Fans over the Ages, Londres.

\section{Eburacum...}

Eburacum. Roman York. An Inventory on the Historical Monuments in the Cily of York, Royal Commission on IIistorical Monuments, England, Londres, 1962.

\section{Greep S.J.}

1983: Objects of Animal Bone, Anller, lvory and Teeth from Roman Britain, Unpublished Ph. D. Thesis, Cniversity College Cardiff.

\section{Liversidge J.}

1973 : Britain in the Roman Empire, Londres, Cardinal. $526 \mathrm{p}$.

\section{Richmond I.}

1946 : The Four Coloniae of Roman Britain, The Archaeological Journal. CIII, p. 79-80. 